Molecules 2006, 11, 603-614

\title{
molecules
}

ISSN 1420-3049

http://www.mdpi.org

Full Paper

\section{Epibatidine Alkaloid Chemistry: 5. Domino-Heck Reactions of Azabicyclic and Tricyclic Systems ${ }^{\dagger}$}

\author{
Çigdem Yolacan ${ }^{1}$, Emine Bagdatli ${ }^{1}$, Nüket Öcal ${ }^{1, *}$ and Dieter E. Kaufmann ${ }^{2, *}$ \\ ${ }^{1}$ Yıldız Technical University, Faculty of Art and Sciences, Department of Chemistry, Davutpasa \\ Campus, 34210, Istanbul, Turkey \\ ${ }^{2}$ Clausthal University of Technology, Institute of Organic Chemistry, Leibnizstr. 6, D-38678 \\ Clausthal-Zellerfeld, Germany \\ ${ }^{\dagger}$ For the preceding paper in this series, see ref. [4] \\ * Authors to whom correspondence may be addressed; e-mails: nocal@yildiz.edu.tr; \\ dieter.kaufmann@tu-clausthal.de
}

Received: 26 July 2006; in revised form: 11 August 2006 / Accepted: 12 August 2006 / Published: 16 August 2006

\begin{abstract}
Palladium-catalyzed hydroarylations and additional domino reactions of azabicyclic and tricyclic norbornene derivatives were investigated and a series of new epibatidine analogues were synthesized.
\end{abstract}

Keywords: Epibatidine, Palladium, Hydroarylation, Domino Reactions, Heck Reactions, Alkynes, Heterocycles.

\section{Introduction}

Epibatidine (1), a novel class of amphibian alkaloid, was first isolated by Daly in trace amounts from the skin of the Ecuadorian poison frog Epipedobates tricolor [1]. The very high analgesic activity of $\mathbf{1}$ is a consequence of its high potency as an agonist towards nicotinic acetylcholine receptors (nAChRs) in the central and autonomic nervous systems [2]. The exciting biological properties and unique structure of $\mathbf{1}$, combined with its scarcity in Nature (ca. $1 \mathrm{mg}$ were obtained from some 750 frogs) have aroused the interest of synthetic chemists [3]. 


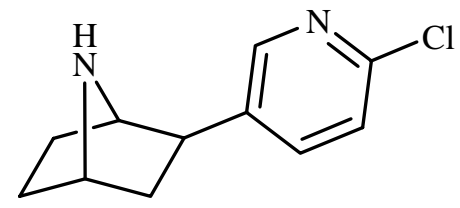

1

Despite the large number of syntheses of epibatidine published to date [4, 5], only a few analogues with modified pyridine rings have been prepared by reductive Heck reactions [6, 7]. In conjuction with this work we became interested in the possibility of synthesizing epibatidine analogues with modified bicyclic ring systems in a single synthetic operation via reductive Heck and additional domino-Heck reactions employing aryl(heteroaryl) iodides.

\section{Results and Discussion}

Our synthesis started with the Hetero-Diels-Alder reaction of cyclopentadiene and the iminium ion generated from formaldehyde and ammonium chloride [8]. The reaction occurred smoothly in aqueous methanol at room temperature to give the bicyclic amine 2. Because of its unstable nature, this secondary amine was protected with benzoyl chloride to provide $\mathbf{3}$ in good yield (Scheme 1).

\section{Scheme 1}

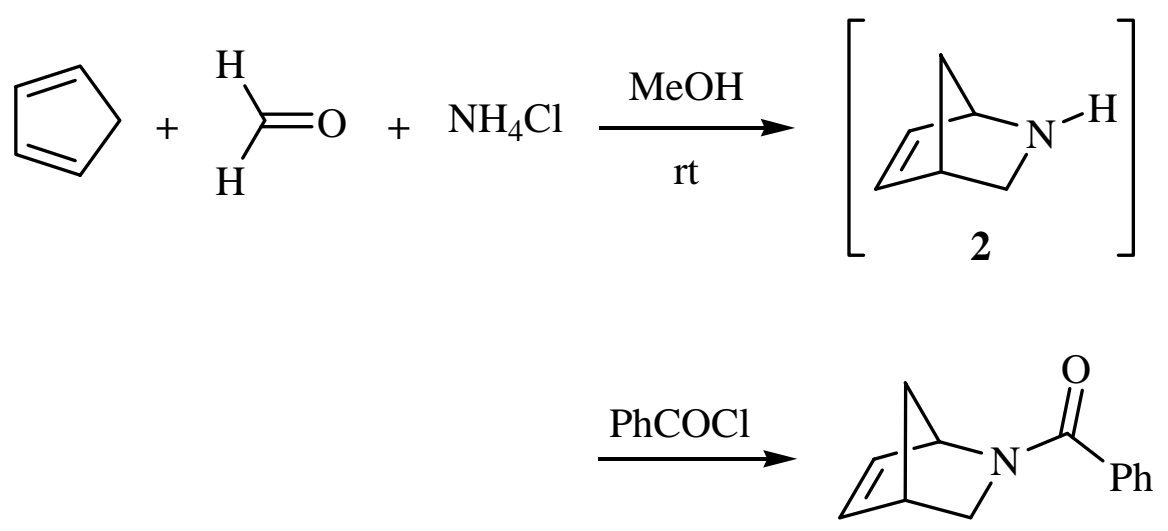

3

Due to the presence of rotamers, all the signals in the NMR spectra of $\mathbf{3}$ appear in pairs. Malpass and co-workers have previously prepared and protected compound $\mathbf{2}$ using both the benzyloxycarbonyl- (Cbz) and t-butoxycarbonyl (BOC) groups. They only worked with 2-chloro-5iodopyridine under reductive Heck conditions using triphenylphosphine $\left(\mathrm{PPh}_{3}\right)$ as the ligand [6]. In our work excellent yields were obtained using triphenylarsine $\left(\mathrm{AsPh}_{3}\right)$ instead of $\mathrm{PPh}_{3}$. Treatment of 3 with iodobenzene, 2-iodothiophene, 2-iodonaphthalene and 2-chloro-5-iodopyridine under reductive Heck conditions gave new compounds $\mathbf{4 a - d}$ and $\mathbf{5 b}$, 5d as regioisomers after chromatographic separation. The reactions with iodobenzene and 2-iodonaphthalene gave only 5-exo- products. The use of trimethylsilylacetylene under domino-Heck conditions provided alkynic bicyclic systems $6 \mathbf{6}$ and $\mathbf{6 f}$ (Scheme 2). The reactivity of the bicyclic double bond in $\mathbf{3}$ is dependent on the nature of the $\mathrm{N}$ protecting group. 


\section{Scheme 2}<smiles>O=C([PH2+])N1CC2C=CC1C2</smiles>

3

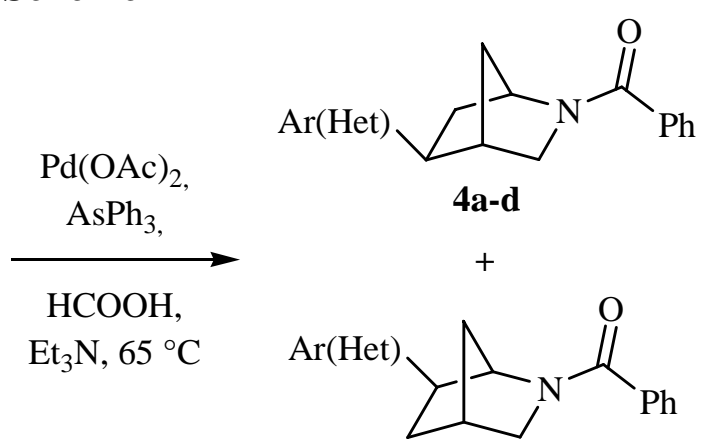

$5 b, 5 d$
$\operatorname{Ar}($ Het $)-=$<smiles>Cc1ccccc1</smiles>

a<smiles>Cc1cccs1</smiles>

b<smiles>Cc1ccc2ccccc2c1</smiles>

c<smiles>Cc1ccc(Cl)nc1</smiles>

d
$\overbrace{\mathrm{N}}^{\mathrm{O}} \overbrace{\mathrm{Ph}}+\mathrm{Ph}-\mathrm{I}+\mathrm{Me}_{3} \mathrm{Si}=$

3

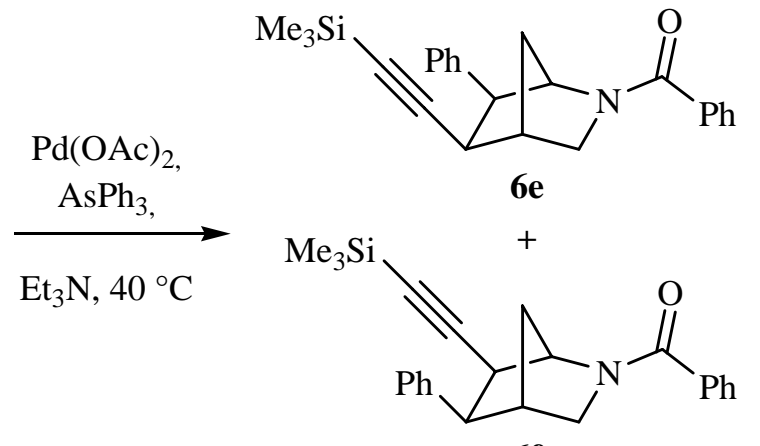

6f

The regiochemistry of new compounds was inferred from their ${ }^{1} \mathrm{H}-\mathrm{NMR}$ and HH-COSY spectra. For example, observation of the $\mathrm{H}_{1}$ proton at $4.15 \mathrm{ppm}$ in the ${ }^{1} \mathrm{H}-\mathrm{NMR}$ spectra of $\mathbf{4 b}$ (red spectrum in Figure 1), while it appeared at $4.04 \mathrm{ppm}$ for $\mathbf{5 b}$ (blue spectrum in Figure 1) was the first evidence for determining both exo-regioisomers. In the HH-COSY spectra of $\mathbf{4 b}$, an interaction between $\mathrm{H}_{6 \mathrm{x}}$ and $\mathrm{H}_{1}$ was seen clearly, but the spectrum of $\mathbf{5 b}$ did not show the same coupling due to the 6-exo- substituent.

Figure 1

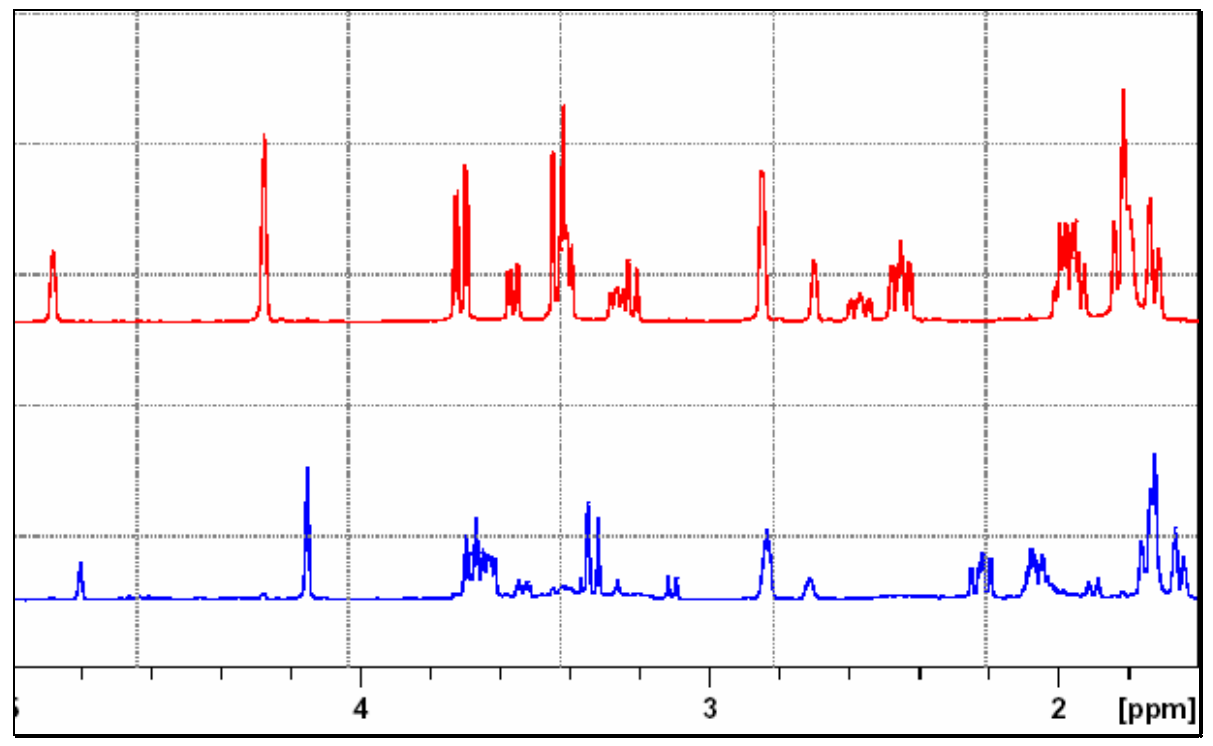


The structures of the regioisomers $6 \mathbf{e}$ and $\mathbf{6 f}$ resulting from the domino-Heck reactions were also identified from the HMBC spectra. In the spectrum of $\mathbf{6 f}$, the interaction of the acetylene carbon (at $103.5 \mathrm{ppm}$ ) with the $\mathrm{H}_{1}$ proton (at $4.24 \mathrm{ppm}$ ) was obvious; in the isomer $\mathbf{6 e}$, an interaction between the phenyl group quaternary carbon (at $139.0 \mathrm{ppm}$ ) with the $\mathrm{H}_{1}$ proton (at $4.40 \mathrm{ppm}$ ) was apparent, but in both cases similar effects were not seen in the other isomers. This provided good evidence for the assignment of the positions of the phenyl and trimethlysilylacetylene substituents on the bicyclic ring.

In the second part of this work we prepared compound 7 by the reaction of $N$-carbomethoxypyrrole with $N$-phenylmaleimide, using the literature procedure [9], to explore tricyclic epibatidine analogues in order to find the most active members of the class (Scheme 3).

\section{Scheme 3}

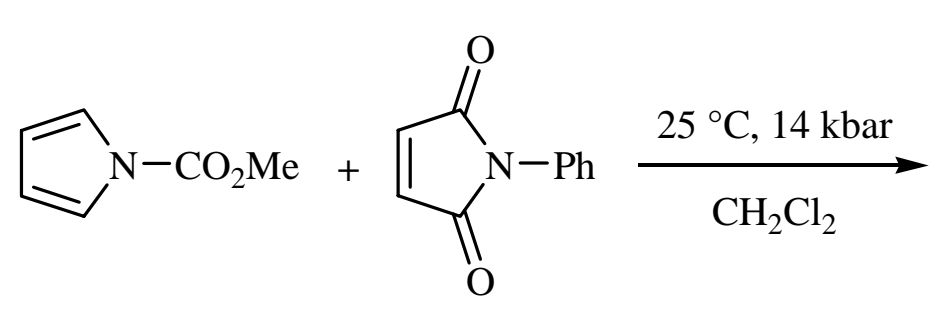

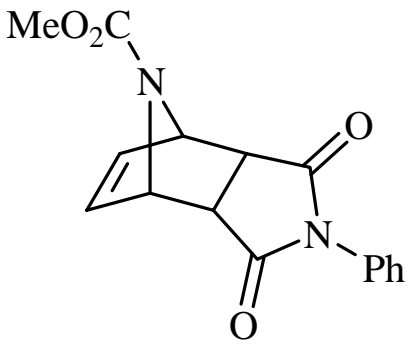

7

Due to the strain in substituted norbornenes and their derivatives such as 7 , the $\mathrm{C}=\mathrm{C}$ double bond reacts readily with many transition metal catalysts. Under typical reductive Heck coupling reaction conditions, and using aryl(heteroaryl) iodides such as iodobenzene, 2-iodothiophene and 4chloroiodobenzene, we obtained a series of bicyclic imides 8a-c as single regioisomers due to the symmetry of $\mathbf{7}$. We also synthesized a new diazatricylic compound 9 using domino-Heck conditions. Not surprisingly, the attachments of phenyl and silylethynyl groups was observed to have taken place exclusively from the exo-side of the bicylic alkene 7 (Scheme 4). The structures of 8a-c and $\mathbf{9}$ were also assigned by analysis of their HH-COSY and HMBC spectra in $\mathrm{CDCl}_{3}$.

\section{Scheme 4}<smiles>COC(=O)N1C2C=CC3C2C(=O)N(c2ccccc2)C(=O)C31</smiles>

7

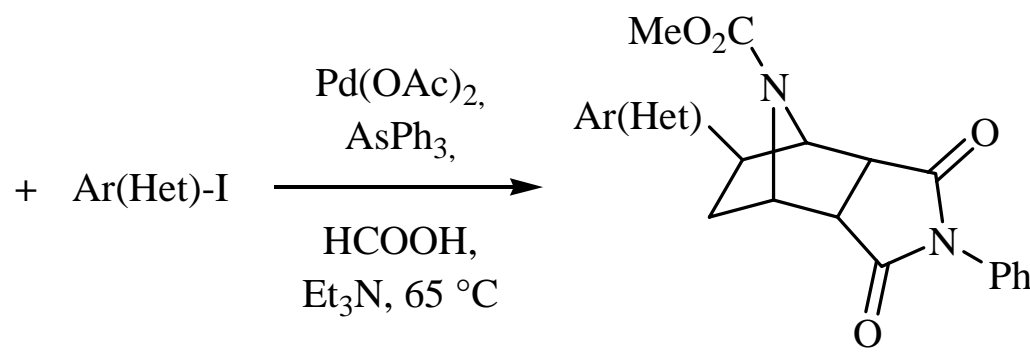

8a-c<smiles>Cc1ccc(Cl)cc1</smiles> 
Scheme 4. Cont.
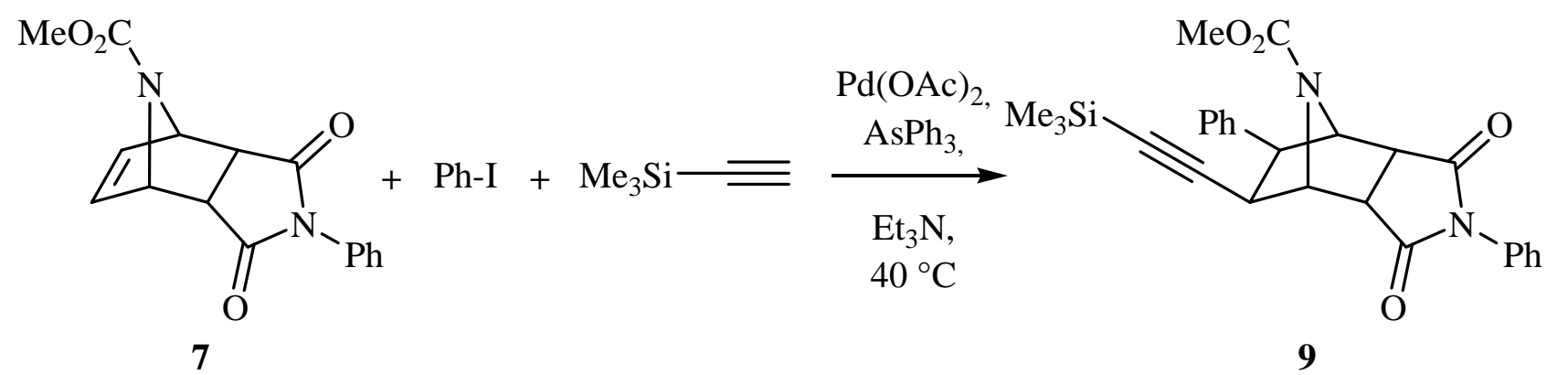

\section{Conclusions}

The palladium-catalyzed hydroarylation of the easily accessible $\mathrm{N}$-benzoylated 2-azabicyclo[2.2.1]heptene (3) in the presence of triphenylarsine as a ligand has been proven to be an excellent, versatile and high-yield approach to aryl- and heteroaryl analogues $\mathbf{4}$ and $\mathbf{5}$ of the bioactive alkaloid epibatidine (1); in case of aryl groups the reaction proceeds regioselectively. Reductive arylations of a diazatricyclic alkene $\mathbf{7}$, synthesized by cycloaddition of a pyrrole carboxylic ester with $N$-phenylmaleimide, also succeeded under comparable reaction conditions. Domino-Heck sequential C-C couplings with aryl halides have been shown to be feasible in the presence of trimethylsilylacetylene. All Heck-type reactions proceed exo-selectively, leading to the same stereochemistry as found in $\mathbf{1}$.

\section{Experimental}

\section{General}

NMR spectra ( $\mathrm{CDCl}_{3}$ solvent) were recorded on Bruker Digital FT-NMR Avance 400 and Varian Inova $500 \mathrm{MHz}$ NMR spectrometers, with TMS as internal reference. In the ${ }^{13} \mathrm{C}$-NMR spectra quaternary, methylene and methyl carbons were identified using DEPT experiments. FTIR spectra $(\mathrm{KBr})$ were recorded on a Perkin Elmer FT-IR spectrometer. GC-EIMS spectra were measured on a Varian SAT2100T/GC3900 spectrometer using ionisation by FAB. Reactions were performed under dry nitrogen. Melting points were measured on a Gallenkamp melting point apparatus. Silicagel 60 (Merck) was used for column chromatography separations. TLC was conducted on standard aluminium sheets pre-coated with a $0.2 \mathrm{~mm}$ layer of silica gel.

\section{Heck Reactions - General procedure}

A solution of palladium(II) acetate [Pd(OAc) 2 ( $5.6 \mathrm{mg}, 25 \mu \mathrm{mol})$ and $\mathrm{AsPh}_{3}(33.7 \mathrm{mg}, 11 \mu \mathrm{mol})$ in anhydrous DMF or DMSO (3 mL) was stirred under nitrogen at $65{ }^{\circ} \mathrm{C}$ for $15 \mathrm{~min}$. Then, compounds 3 or $7(1.0 \mathrm{mmol}), \mathrm{Et}_{3} \mathrm{~N}(488 \mu \mathrm{L}, 3.5 \mathrm{mmol})$, the appropriate aryl(heteroaryl) iodide (1.5 mmol) and $\mathrm{HCOOH}$ (138 mg, $3.0 \mathrm{mmol}$ ) were added. The reaction mixture was stirred for 8-24 h. After cooling to r.t. EtOAc and brine added, the organic layer was separated, dried $\left(\mathrm{MgSO}_{4}\right)$, filtered and the solvent evaporated. The residue was purified by column chromatography. 


\section{Domino-Heck Reactions - General Procedure}

$\mathrm{Pd}(\mathrm{OAc})_{2}$ (5.6 mg, $\left.25 \mu \mathrm{mol}\right)$ and the arsine ligand (55 $\left.\mu \mathrm{mol}\right)$ were dissolved in dry DMF (3 mL) and the solution was stirred at $40{ }^{\circ} \mathrm{C}$ for $15 \mathrm{~min}$. Then, 3 or 7 (1.0 mmol), the aryl compound (1.5 mmol), triethylamine $(488 \mu \mathrm{L}, 3.50 \mathrm{mmol})$ and trimethylsilylacetylene $(3.00 \mathrm{mmol})$ were added rapidly in one portion. The mixture was heated at the same temperature for $24 \mathrm{~h}$. After cooling down to r.t. brine $(50 \mathrm{~mL})$ was added, the reaction mixture was extracted with ethyl acetate and dried over $\mathrm{MgSO}_{4}$. The solvent was evaporated and the residue purified by column chromatography.

N-Benzoyl-2-azabicyclo[2.2.1]hept-5-ene (3)

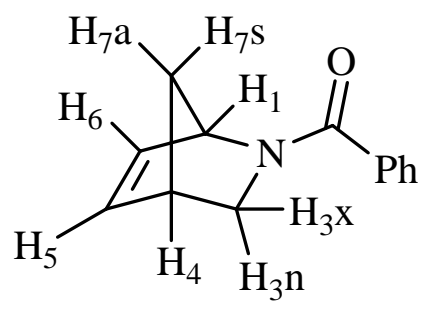

Freshly distilled cyclopentadiene (4 mL, $50 \mathrm{mmol}$ ) was added to a solution of $\mathrm{NH}_{4} \mathrm{Cl}$ (1.325 g, 25 $\mathrm{mmol}), 36 \%$ aqueous formaldehyde $(2.6 \mathrm{~mL}, 35 \mathrm{mmol})$ and $\mathrm{MeOH}(5 \mathrm{~mL})$. The reaction mixture was stirred overnight at r.t. The resulting light yellow solution was diluted with an equal volume of water and washed with diethyl ether $(2 \times 15 \mathrm{~mL})$. To a mixture of this extract and 10\% $\mathrm{NaOH}(5 \mathrm{~mL})$ benzoyl chloride (2.10 g, $10 \mathrm{mmol}$ ) was added over $10 \mathrm{~min}$ at r.t. and the mixture was allowed to stir for $2 \mathrm{~h}$. The organic layer was separated, dried over $\mathrm{Na}_{2} \mathrm{SO}_{4}$, filtered and the solvent removed under reduced pressure to give a yellow oil that was separated by column chromatography (1:1 ethyl acetate- $n$ hexane) to give 3 in $83 \%$ yield; colorless crystals; $\mathrm{R}_{\mathrm{f}}$ : 0.57; mp $49-51{ }^{\circ} \mathrm{C}$; IR: $3060,1622,1575,1495$, 1426, 1176, 710, $659 \mathrm{~cm}^{-1}$; ${ }^{1} \mathrm{H}-\mathrm{NMR}$ (rotamer ratio= 1:0.6) $\delta: 1.56-1.62$ (dd, J=8.0, $6.5 \mathrm{~Hz}, 4 \mathrm{H}, \mathrm{H}_{7 \mathrm{a}}$ and $\mathrm{H}_{7 \mathrm{~s}}$ ), [2.54-2.56 (dd, $J=1.5,1.5 \mathrm{~Hz}$, minor rotamer) and 2.90-2.92 (dd, $J=1.5,1.5 \mathrm{~Hz}$, major rotamer), $2 \mathrm{H}, \mathrm{H}_{3 \mathrm{n}}$ ], [3.14 (bs, minor rotamer) and 3.24 (bs, major rotamer), 2H, $\mathrm{H}_{4}$ ], 3.49-3.55 (m, major and minor rotamers, $2 \mathrm{H}, \mathrm{H}_{3 \mathrm{x}}$ ), [4.46 (bs, major rotamer) and 5.14 (bs, minor rotamer), $2 \mathrm{H}, \mathrm{H}_{1}$ ], [6.18-6.20 (dd, $J=2.0,2.0 \mathrm{~Hz}$, minor rotamer) and 6.20-6.22 (dd, $J=2.0,2.0 \mathrm{~Hz}$, major rotamer, $2 \mathrm{H}$, $\mathrm{H}_{6}$ )], [6.34-6.36 (dd, $J=2.0,2.0 \mathrm{~Hz}$, major rotamer) and 6.48-6.50 (dd, $J=2.0,2.0 \mathrm{~Hz}$, minor rotamer), $2 \mathrm{H}, \mathrm{H}_{5}$ ], 7.30-7.45 (m, 10H, aromatic protons); ${ }^{13} \mathrm{C}-\mathrm{NMR} \delta: 42.8,45.9,49.2,64.0,127.3,128.5,128.6$, 133.4, 137.3, 138.5, 171.3 (major rotamer); 44.0, 47.6, 49.8, 60.5, 127.6, 128.4, 130.3, 133.2, 135.9, 136.6, 169.5 (minor rotamer); MS: m/z $199\left[\mathrm{M}^{+}\right], \mathrm{C}_{13} \mathrm{H}_{13} \mathrm{NO}$.

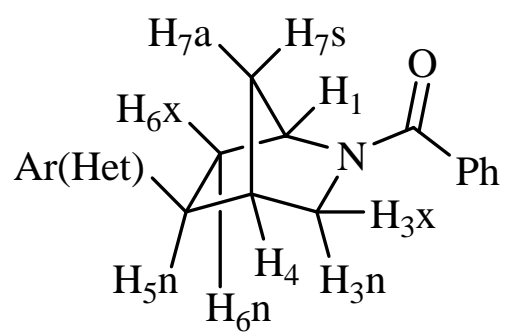

4a-d

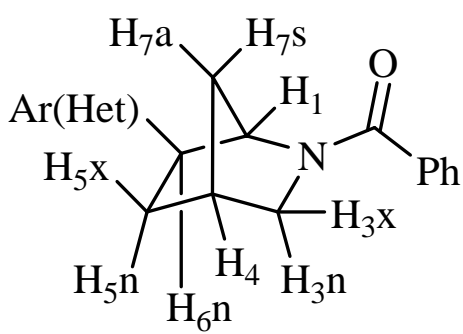

5a, 5d 
N-Benzoyl-5-exo-phenyl-2-azabicyclo[2.2.1]heptane (4a).

Purified by column chromatography (1:1 ethyl acetate- $n$-hexane) as colorless crystals in $91 \%$ yield; $\mathrm{R}_{\mathrm{f}}$ : 0.42 ; mp 81-83 ${ }^{\circ} \mathrm{C}$; IR: 3030, 1622, 1575, 1494, 1423, 1195, 710, $653 \mathrm{~cm}^{-1}$; ${ }^{1} \mathrm{H}-\mathrm{NMR}$ (rotamer ratio=1:0.5) $\delta$ : 1.51-1.70 (m, major and minor rotamers, $4 \mathrm{H}, \mathrm{H}_{7 \mathrm{a}}$ and $\mathrm{H}_{7 \mathrm{~s}}$ ), [1.76-1.86 (m, major rotamer) and 1.92-2.20 (m, minor rotamer), $2 \mathrm{H}, \mathrm{H}_{6 \mathrm{x}}$ ], [2.26-2.32 (ddt, $J=2.0,2.5 \mathrm{~Hz}$, major rotamer) and 2.38-2.45 (ddt, $J=2.5,2.5 \mathrm{~Hz}$, minor rotamer), $2 \mathrm{H}, \mathrm{H}_{6 \mathrm{n}}$ ], [2.60 (bs, minor rotamer) and 2.74 (bs, major rotamer), $\left.2 \mathrm{H}, \mathrm{H}_{4}\right]$, [2.90-2.96 (m, minor rotamer) and 3.06-3.14 (m, major rotamer), $2 \mathrm{H}, \mathrm{H}_{5 \mathrm{n}}$ ], [3.24-3.28 (m, minor rotamer) and 3.32-3.36 (m, major rotamer), $2 \mathrm{H}, \mathrm{H}_{3 \mathrm{n}}$ ], [3.40-3.48 (m, minor rotamer) and 3.54-3.64 (m, major rotamer), $\left.2 \mathrm{H}, \mathrm{H}_{3 \mathrm{x}}\right]$, [4.08 (bs, major rotamer) and 4.74 (bs, minor rotamer), $2 \mathrm{H}, \mathrm{H}_{1}$ ], [7.08-7.27 (m, major and minor rotamers), 10H, aromatic protons], [7.30-7.51 (m, major and minor rotamers), $10 \mathrm{H}$, aromatic protons]; ${ }^{13} \mathrm{C}-\mathrm{NMR} \delta$ : 35.2, 36.4, 40.6, 42.6, 53.5, 57.4, 126.6, 126.9, 127.2, 127.4, 128.6, 128.8, 130.1, 137.1, 144.9, 169.2 (major rotamer); 35.0, 35.7, 39.0, 44.1, 52.4, 56.9, 126.4, 127.0, 127.1, 127.4, 128.5, 128.8, 130.1, 135.4, 142.5, 169.6 (minor rotamer); MS: m/z $277\left[\mathrm{M}^{+}\right], \mathrm{C}_{19} \mathrm{H}_{19} \mathrm{NO}$.

N-Benzoyl-5-exo-(2-thienyl)-2-azabicyclo[2.2.1 heptane (4b).

Separated by column chromatography (4:1 ethyl acetate- $n$-hexane) as a yellow oil, yield $57 \%$; $\mathrm{R}_{\mathrm{f}}$ : 0.67; IR: 3028, 1624, 1575, 1424, 1197, 798, $700 \mathrm{~cm}^{-1}$; ${ }^{1} \mathrm{H}-\mathrm{NMR}$ (rotamer ratio=1:0.4) $\delta: 1.58-1.74$ (m, major and minor rotamers, $4 \mathrm{H}, \mathrm{H}_{7 \mathrm{a}}$ and $\mathrm{H}_{7 \mathrm{~s}}$ ), 1.80-1.89 (m, major and minor rotamers, $2 \mathrm{H}, \mathrm{H}_{6 \mathrm{x}}$ ), [2.30-2.36 (ddt, $J=2.5,2.5 \mathrm{~Hz}$, major rotamer) and 2.42-2.48 (ddt, $J=2.0,2.0 \mathrm{~Hz}$, minor rotamer), $2 \mathrm{H}$, $\mathrm{H}_{6 \mathrm{n}}$ ], [2.58 (bs, minor rotamer) and 2.72 (bs, major rotamer), $2 \mathrm{H}, \mathrm{H}_{4}$ ], 3.13-3.19 (m, major and minor rotamers, $2 \mathrm{H}, \mathrm{H}_{5 \mathrm{n}}$ ), 3.23-3.34 (m, major and minor rotamers, $2 \mathrm{H}, \mathrm{H}_{3 \mathrm{n}}$ ], [3.41-3.46 (dd, $J=3.5,3.0 \mathrm{~Hz}$ minor rotamer) and 3.59-3.62 (dd, $J=3.5,3.5 \mathrm{~Hz}$, major rotamer), $2 \mathrm{H}, \mathrm{H}_{3 \mathrm{x}}$ ], [4.15 (bs, major rotamer) and 4.74 (bs, minor rotamer), $2 \mathrm{H}, \mathrm{H}_{1}$ ], 6.72-6.75 (m, major and minor rotamers, $2 \mathrm{H}$, thienyl protons), 6.83-6.88 (m, major and minor rotamers, $2 \mathrm{H}$, thienyl protons), 7.04-7.08 (m, major and minor rotamers, $2 \mathrm{H}$, thienyl protons), 7.30-7.46 ( $\mathrm{m}$, major and minor rotamers, $10 \mathrm{H}$, aromatic protons); ${ }^{13} \mathrm{C}-\mathrm{NMR} \delta$ : 36.2, 41.3, 41.8, 44.4, 52.5, 59.8, 123.1, 123.3, 126.8, 127.1, 128.4, 130.0, 136.6, 149.3, 169.0 (major rotamer); 34.8, 40.3, 41.1, 45.7, 54.5, 56.7, 123.1, 123.3, 126.8, 127.2, 128.3, 130.1, 136.5, 149.5, 169.7 (minor rotamer); MS: m/z $283\left[\mathrm{M}^{+}\right], \mathrm{C}_{17} \mathrm{H}_{17} \mathrm{NOS}$.

N-Benzoyl-5-exo-(2-naphthyl)-2-azabicyclo[2.2.1 Jheptane (4c).

Separated by column chromatography (1:1 ethyl acetate- $n$-hexane) as white crystals, yield $96 \%$; $\mathrm{R}_{\mathrm{f}}$ : 0.42; mp 56-58 ${ }^{\circ} \mathrm{C}$; IR: 3034, 1625, 1574, 1508, 1260, 779, $701 \mathrm{~cm}^{-1} ;{ }^{1} \mathrm{H}-\mathrm{NMR}$ (rotamer ratio=1:0.8) $\delta$ : 1.71-1.92 (m, major and minor rotamers, $4 \mathrm{H}, \mathrm{H}_{7 \mathrm{a}}$ and $\mathrm{H}_{7 \mathrm{~s}}$ ), [1.93-2.01 (m, major rotamer) and 2.032.12 (m, minor rotamer), $2 \mathrm{H}, \mathrm{H}_{6 \mathrm{x}}$ ], [2.29-2.35 (dddd, $J=1.5,2.0,2.0,3.0 \mathrm{~Hz}$, major rotamer) and 2.572.64 (dddd, $J=2.0,2.5,2.5,3.0 \mathrm{~Hz}$, minor rotamer), $2 \mathrm{H}, \mathrm{H}_{6 \mathrm{n}}$ ], [2.83 (bs, minor rotamer) and 2.90 (bs, major rotamer), $2 \mathrm{H}, \mathrm{H}_{4}$ ], [3.18-3.22 (d, $J=9.5 \mathrm{~Hz}$, minor rotamer) and 3.34-3.38 (d, $J=9.0 \mathrm{~Hz}$, major rotamer), $2 \mathrm{H}, \mathrm{H}_{5 \mathrm{n}}$ ], [3.48-3.52 (dd, $J=1.0,1.0 \mathrm{~Hz}$, minor rotamer) and 3.56-3.60 (dd, $J=1.0,1.5 \mathrm{~Hz}$ major rotamer) $\left.2 \mathrm{H}, \mathrm{H}_{3 \mathrm{n}}\right]$, [3.65-3.72 (m, minor rotamer) and 3.76-3.82 (m, major rotamer), $\left.2 \mathrm{H}, \mathrm{H}_{3 \mathrm{x}}\right]$, 
[4.55 (bs, major rotamer) and 5.01 (bs, minor rotamer), 2H, $\mathrm{H}_{1}$ ], 7.11-7.20 (d, $J=7.0 \mathrm{~Hz}$, major rotamer, $1 \mathrm{H}$, aromatic proton), 7.28-7.47 (m, major and minor rotamers, $13 \mathrm{H}$, aromatic protons), 7.48-7.62 (m, major and minor rotamers, $4 \mathrm{H}$ aromatic protons), 7.66-7.70 (d, $J=9.5 \mathrm{~Hz}$, minor rotamer, $1 \mathrm{H}$, aromatic proton), 7.71-7.74 (d, $J=8.0 \mathrm{~Hz}$, major rotamer, $1 \mathrm{H}$, aromatic proton), 7.80-7.83 (d, $J=8.5 \mathrm{~Hz}$, minor rotamer, $1 \mathrm{H}$, aromatic proton), 7.84-7.88 (d, $J=8.0 \mathrm{~Hz}$, major rotamer, $1 \mathrm{H}$, aromatic proton), 7.98-8.02 (d, $J=7.5 \mathrm{~Hz}$, minor rotamer, $1 \mathrm{H}$, aromatic proton), 8.04-8.08 (d, $J=9.0 \mathrm{~Hz}$, major rotamer, $1 \mathrm{H}$, aromatic proton); ${ }^{13} \mathrm{C}-\mathrm{NMR} \delta$ : 35.1, 37.0, 39.4, 41.4, 53.5, 60.5, 121.4, 124.0, 125.5, 125.9, 126.3, 127.3, 127.5, 128.7, 129.2, 130.2, 131.8, 134.2, 137.0, 140.6, 169.2 (major rotamer); 34.7, 37.0, 39.7, 41.3, 52.9, 60.9, 122.1, 123.7, 125.3, 125.9, 126.3, 127.2, 127.4, 128.6, 129.0, 130.2, 131.7, 134.4, 136.9, 141.0, 169.0 (minor rotamer); MS: m/z $327\left[\mathrm{M}^{+}\right], \mathrm{C}_{23} \mathrm{H}_{21} \mathrm{NO}$.

N-Benzoyl-5-exo-(2-chloro-5-pyridinyl)-2-azabicyclo[2.2.1 Jheptane (4d).

Separated by column chromatography (1:1 ethyl acetate- $n$-hexane) as a colorless oil, yield 54\%; $\mathrm{R}_{\mathrm{f}}$ : 0.45; IR: 3065, 1627, 1574, 1508, 1260, 780, $714 \mathrm{~cm}^{-1}$; ${ }^{1} \mathrm{H}-\mathrm{NMR}$ (rotamer ratio=1:0.6) $\delta: 1.52-1.70$ (m, major and minor rotamers, $4 \mathrm{H}, \mathrm{H}_{7 \mathrm{a}}$ and $\mathrm{H}_{7 \mathrm{~s}}$ ), [1.78-2.20 (m, major and minor rotamers), $2 \mathrm{H}, \mathrm{H}_{6 \mathrm{x}}$ ], [2.28-2.32 (dddd, $J=2.0,2.0,2.5,2.5 \mathrm{~Hz}$, major rotamer) and 2.38-2.44 (dddd, $J=2.0,2.0,2.5,2.5 \mathrm{~Hz}$, minor rotamer), $2 \mathrm{H}, \mathrm{H}_{6 \mathrm{n}}$ ], [2.62 (bs, minor rotamer) and 2.74 (bs, major rotamer), $2 \mathrm{H}, \mathrm{H}_{4}$ ], [2.90-3.00 (m, minor rotamer) and 3.06-3.15 (m, major rotamer), $\left.2 \mathrm{H}, \mathrm{H}_{5 \mathrm{n}}\right]$, [3.22-3.28 (m, minor rotamer) and 3.33-3.38 (m, major rotamer) $\left.2 \mathrm{H}, \mathrm{H}_{3 \mathrm{n}}\right],[3.43-3.48(\mathrm{~m}$, minor rotamer) and 3.52-3.62 (m, major rotamer), $2 \mathrm{H}, \mathrm{H}_{3 \mathrm{x}}$ ], [4.14 (bs, major rotamer) and 4.78 (bs, minor rotamer), 2H, $\mathrm{H}_{1}$ ], 7.26-7.28 (d, $J=8.0 \mathrm{~Hz}$, major and minor rotamers, $2 \mathrm{H}$, aromatic protons), 7.30-7.47 (m, major and minor rotamers, $10 \mathrm{H}$, aromatic protons), 7.47-7.49 (dd, $J=8.0,3.0 \mathrm{~Hz}, 2 \mathrm{H}$, major and minor rotamers, aromatic protons), 8.22-8.24 (d, $J=3.0 \mathrm{~Hz}, 2 \mathrm{H}$, major and minor rotamers, aromatic protons); ${ }^{13} \mathrm{C}-\mathrm{NMR} \delta$ : 35.1 , 36.4, 40.5, 42.7, 53.4, 57.5, 126.6, 126.9, 127.1, 128.5, 128.7, 130.0, 136.7, 139.2, 148.4, 169.1 (major rotamer), 35.0, 36.2, 40.8, 42.9, 54.0, 57.9, 127.0, 127.2, 128.9, 129.0, 130.5, 137.0, 139.8, 148.7, 170.2 (minor rotamer); MS: m/z $312\left[\mathrm{M}^{+}\right], \mathrm{C}_{18} \mathrm{H}_{17} \mathrm{ClN}_{2} \mathrm{O}$.

\section{N-Benzoyl-6-exo-(2-thienyl)-2-azabicyclo[2.2.1 heptane (5b).}

Separated by column chromatography (4:1 ethyl acetate- $n$-hexane) as yellow crystals, yield 40\%; $\mathrm{R}_{\mathrm{f}}$ : 0.60; mp 98-100 ${ }^{\circ} \mathrm{C}$, IR: 3025, 1613, 1571, 1430, 1074, 789, $716 \mathrm{~cm}^{-1}$; ${ }^{1} \mathrm{H}-\mathrm{NMR}$ (rotamer ratio= 1:0.3) $\delta$ : 1.52-1.66 (m, major and minor rotamers, $4 \mathrm{H}, \mathrm{H}_{7 \mathrm{a}}$ and $\mathrm{H}_{7 \mathrm{~s}}$ ), 1.92-1.98 (m, major and minor rotamers, $2 \mathrm{H}, \mathrm{H}_{5 \mathrm{x}}$ ), 2.08-2.14 (m, major and minor rotamers, $2 \mathrm{H}, \mathrm{H}_{5 \mathrm{n}}$ ), [2.60 (bs, minor rotamer) and 2.72 (bs, major rotamer), $2 \mathrm{H}, \mathrm{H}_{4}$ ], [3.08-3.11 (dd, $J=1.0,1.0 \mathrm{~Hz}$, minor rotamer) and 3.20-3.23 (dd, $J=1.5,1.5 \mathrm{~Hz}$ major rotamer), $2 \mathrm{H}, \mathrm{H}_{3 \mathrm{n}}$ ], [3.40-3.43 (tt, minor rotamer) and 3.49-3.52 (m, major rotamer), $2 \mathrm{H}, \mathrm{H}_{3 \mathrm{x}}$ ], 3.54-3.60 (m, major and minor rotamers, $2 \mathrm{H}, \mathrm{H}_{6 \mathrm{n}}$ ), [4.04 (bs, major rotamer) and 4.70 (bs, minor rotamer), $2 \mathrm{H}, \mathrm{H}_{1}$ ], 6.82-6.90 (dd, $J=1.0,1.0 \mathrm{~Hz}$, major and minor rotamers, $2 \mathrm{H}$, thienyl protons), 7.04-7.10 (dd, $J=1.0,1.0 \mathrm{~Hz}$, major and minor rotamers, 4H, thienyl protons), 7.28-7.52 (m, major and minor rotamers, $10 \mathrm{H}$, aromatic protons); ${ }^{13} \mathrm{C}-\mathrm{NMR} \delta$ : 35.7, 37.1, 39.2, 45.0, 52.1, 65.8, 123.4, 123.6, 127.0, 127.1, 128.4, 130.2, 136.2, 146.6, 168.9 (major rotamer), 36.2, 37.5, 39.5, 43.1, 
56.9, 62.2, 123.3, 123.8, 126.9, 127.3, 128.3, 130.2, 136.6, 147.6, 169.5 (minor rotamer); MS: m/z $283\left[\mathrm{M}^{+}\right], \mathrm{C}_{17} \mathrm{H}_{17} \mathrm{NOS}$.

\section{N-Benzoyl-6-exo-(2-chloro-5-pyridinyl)-2-azabicyclo[2.2.1 hheptane (5d).}

Separated by column chromatography (1:1 ethyl acetate- $n$-hexane) as a colorless oil, yield $40 \%$; $\mathrm{R}_{\mathrm{f}}$ : 0.39; IR: 3065, 1625, 1575, 1510, 1260, 781, $712 \mathrm{~cm}^{-1}$; ${ }^{1} \mathrm{H}-\mathrm{NMR}$ (rotamer ratio=1:0.55) $\delta$ : 1.52-1.68 (m, major and minor rotamers, $4 \mathrm{H}, \mathrm{H}_{7 \mathrm{a}}$ and $\mathrm{H}_{7 \mathrm{~s}}$ ), [1.92-2.00 (m, major rotamer and minor rotamers), $2 \mathrm{H}, \mathrm{H}_{5 \mathrm{x}}$ ], [2.02-2.12 (m, major rotamer and minor rotamers), $2 \mathrm{H}, \mathrm{H}_{5 \mathrm{n}}$ ], [2.60 (bs, minor rotamer) and 2.74 (bs, major rotamer), $\left.2 \mathrm{H}, \mathrm{H}_{4}\right]$, [3.08-3.13 (dd, $J=1.0,1.0 \mathrm{~Hz}$, minor rotamer) and 3.19-3.24 (dd, $J=1.5,1.5 \mathrm{~Hz}$, major rotamer), 2H, $\mathrm{H}_{3 \mathrm{n}}$ ], [3.40-3.45 (tt, minor rotamer) and 3.48-3.52 (tt, major rotamer) $2 \mathrm{H}, \mathrm{H}_{3 \mathrm{x}}$ ], 3.54-3.58 (m, major rotamer and minor rotamers), $2 \mathrm{H}, \mathrm{H}_{6 \mathrm{n}}$ ], [4.16 (bs, major rotamer) and 4.72 (bs, minor rotamer), $\left.2 \mathrm{H}, \mathrm{H}_{1}\right], 7.20-7.40(\mathrm{~m}$, major and minor rotamers, $12 \mathrm{H}$, aromatic protons), 7.41-7.50 (dd, $J=8.5,2.5 \mathrm{~Hz}$, major and minor rotamers, $2 \mathrm{H}$, aromatic protons), 8.16-8.18 (d, $J=2.5 \mathrm{~Hz}$, major and minor rotamers, $2 \mathrm{H}$, aromatic protons); ${ }^{13} \mathrm{C}-\mathrm{NMR} \delta$ : $35.2,36.5$, 37.2, 45.1, 52.0, 62.0, 126.8, 127.8, 128.1, 128.6, 130.1, 136.9, 145.3, 149.4, 169.7 (major rotamer), 35.4, 36.8, 37.8, 45.7, 52.3, 61.8, 127.0, 128.0, 128.2, 128.9, 131.0, 137.4, 145.6, 149.8, 170.0 (minor rotamer); MS: m/z $312\left[\mathrm{M}^{+}\right], \mathrm{C}_{18} \mathrm{H}_{17} \mathrm{ClN}_{2} \mathrm{O}$.

\section{N-Benzoyl-5-exo-(trimethylsilylethynyl)-6-exo-phenyl-2-azabicyclo[2.2.1 Jheptane (6e).}

Separated by column chromatography (2:3 ethyl acetate- $n$-hexane) as yellow crystals, yield $60 \%$; $\mathrm{R}_{\mathrm{f}}$ : 0.38; mp 113-115 ${ }^{\circ} \mathrm{C}$; IR: 3025, 2177, 1629, 1574, 1427, 1085, 840, $696 \mathrm{~cm}^{-1}$; ${ }^{1} \mathrm{H}-\mathrm{NMR}$ (rotamer ratio=1:0.4) $\delta$ : -0.154 (s, major rotamer, $\left.9 \mathrm{H}, \mathrm{Si}\left(\mathrm{CH}_{3}\right)_{3}\right),-0.145$ (s, minor rotamer, $\left.9 \mathrm{H}, \mathrm{Si}\left(\mathrm{CH}_{3}\right)_{3}\right)$, 1.811.89 (m, major and minor rotamers, $4 \mathrm{H}, \mathrm{H}_{7 \mathrm{a}}$ and $\mathrm{H}_{7 \mathrm{~s}}$ ), [2.19-2.22 (d, $J=10.4 \mathrm{~Hz}$, major rotamer) and 2.29-2.31 (d, $J=10.8 \mathrm{~Hz}$, minor rotamer), $2 \mathrm{H}, \mathrm{H}_{6 \mathrm{n}}$ ], [2.78 (bs, minor rotamer) and 2.89 (bs, major rotamer), $2 \mathrm{H}, \mathrm{H}_{4}$ ], [3.11-3.13 (m, minor rotamer) and 3.24-3.31 (m, major rotamer), $2 \mathrm{H}, \mathrm{H}_{5 \mathrm{n}}$ ], 3.463.50 (dd, $J=4.0,3.6 \mathrm{~Hz}$, minor rotamer, $1 \mathrm{H}, \mathrm{H}_{3 \mathrm{n}}$ ), [3.69-3.72 (dd, $J=4.0,4.0 \mathrm{~Hz}$, major rotamer) and 3.51-3.53 (d, $J=9.6$, minor rotamer), $2 \mathrm{H}, \mathrm{H}_{3 \mathrm{x}}$ ], 3.55-3.57 (d, $J=9.2 \mathrm{~Hz}$, major rotamer, $1 \mathrm{H}, \mathrm{H}_{3 \mathrm{n}}$ ), [4.40 (bs, major rotamer) and 5.05 (bs, minor rotamer), $\left.2 \mathrm{H}, \mathrm{H}_{1}\right], 7.06-7.28$ (m, major and minor rotamers, $10 \mathrm{H}$, aromatic protons), 7.29-7.54 (m, major and minor rotamers, $10 \mathrm{H}$, aromatic protons); ${ }^{13} \mathrm{C}-\mathrm{NMR} \delta$ : -0.42, 36.4, 40.9, 43.6, 50.9, 53.5, 62.6, 89.6, 106.0, 126.4, 127.1, 127.9, 128.0, 128.4, 130.0, 136.0, 139.0, 168.3 (major rotamer), -0.51, 34.9, 40.6, 44.9, 51.9, 54.5, 58.8, 89.6, 106.3, 126.2, 127.2, 127.7,

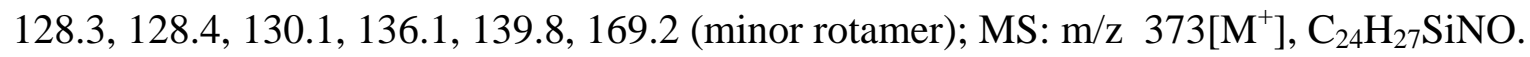

N-Benzoyl-6-exo-(trimethylsilylethynyl)-exo-5-phenyl-2-azabicyclo[2.2.1 heptane (6f).

Separated by column chromatography (2:3 ethyl acetate- $n$-hexane) as yellow crystals, yield 35\%; $\mathrm{R}_{\mathrm{f}}$ : 0.43; mp 128-130 ${ }^{\circ} \mathrm{C}$; IR: 3026, 2171, 1626, 1573, 1418, 1072, 835, $695 \mathrm{~cm}^{-1}$; ${ }^{1} \mathrm{H}-\mathrm{NMR}$ (rotamer ratio=1:0.4) $\delta$ : -0.199 (s, major rotamer, $\left.9 \mathrm{H}, \mathrm{Si}\left(\mathrm{CH}_{3}\right)_{3}\right)$, -0.147 (s, minor rotamer, $\left.9 \mathrm{H}, \mathrm{Si}\left(\mathrm{CH}_{3}\right)_{3}\right)$, [1.841.86 (m, major rotamer) and 1.90-1.93 (m, minor rotamer), 4H, $\mathrm{H}_{7 \mathrm{a}}$ and $\mathrm{H}_{7 \mathrm{~s}}$ ), [2.26-2.29 (d, $J=10.0 \mathrm{~Hz}$, major rotamer) and 2.37-2.39 (d, $J=10.4 \mathrm{~Hz}$, minor rotamer), $2 \mathrm{H}, \mathrm{H}_{6 \mathrm{n}}$ ], [2.83 (bs, minor rotamer) and 
2.98 (bs, major rotamer), $2 \mathrm{H}, \mathrm{H}_{4}$ ], 3.13-3.16 (m, major and minor rotamers, $2 \mathrm{H}, \mathrm{H}_{5 \mathrm{n}}$ ), 3.37-3.41 (m, major and minor rotamers, $2 \mathrm{H}, \mathrm{H}_{3 \mathrm{n}}$ ), [3.56-3.59 (dd, $J=3.6,3.2 \mathrm{~Hz}$, minor rotamer) and 3.64-3.68 (dd, $J=3.6,3.2 \mathrm{~Hz}$, major rotamer), $2 \mathrm{H}, \mathrm{H}_{3 \mathrm{x}}$ ], [4.24 (bs, major rotamer) and 4.78 (bs, minor rotamer), $2 \mathrm{H}$, $\mathrm{H} 1$ ], 7.15-7.25 (m, major and minor rotamers, 5H, aromatic protons), 7.26-7.31 (m, major and minor rotamers, $4 \mathrm{H}$, aromatics), 7.40-7.48 (m, major and minor rotamers, 5H, aromatic protons), 7.52-7.56 (m, major and minor rotamer, $6 \mathrm{H}$, aromatic protons); ${ }^{13} \mathrm{C}-\mathrm{NMR} \delta$ : $-0.51,36.9,40.2,44.7,50.2,54.0$, 64.4, 91.2, 103.5, 126.3, 127.1, 127.9, 128.0, 128.5, 130.1, 136.1, 140.9, 169.3 (major rotamer), -0.43, 34.8, 41.8, 43.0, 49.6, 57.8, 61.1, 90.6, 104.1, 126.2, 127.2, 127.8, 128.0, 128.3, 130.2, 136.1, 141.1, 169.8 (minor rotamer); MS: m/z $373\left[\mathrm{M}^{+}\right], \mathrm{C}_{24} \mathrm{H}_{27} \mathrm{SiNO}$.

endo-Methyl 3,5-dioxo-4-phenyl-4,10-diazatricyclo[5.2.1.0 2,6]dec-8-ene-10-carboxylate (7).

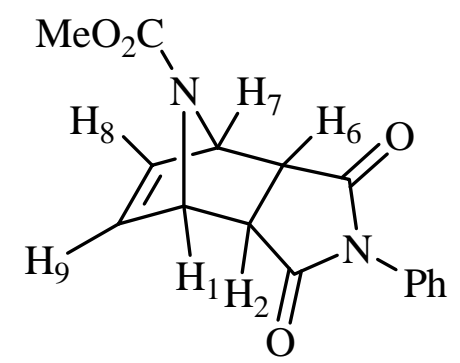

IR: 3065, 1774, 1713, $1697 \mathrm{~cm}^{-1}$; ${ }^{1} \mathrm{H}-\mathrm{NMR} \delta: 2.96$ (s, 2H, $\mathrm{H}_{2}$ and $\mathrm{H}_{6}$ ), 3.62 (s, 3H, $\left.-\mathrm{OCH}_{3}\right), 5.21$ (s, $2 \mathrm{H}, \mathrm{H}_{1}$ and $\mathrm{H}_{7}$ ), 6.58 (s, $2 \mathrm{H}, \mathrm{H}_{8}$ and $\mathrm{H}_{9}$ ), 7.24-7.48 (m, 5H, aromatic protons); ${ }^{13} \mathrm{C}-\mathrm{NMR} \delta$ : 53.1, 63.5, 126.3, 128.8, 129.1, 131.5, 156.0, 174.5,175.1; MS: m/z $298\left[\mathrm{M}^{+}\right], \mathrm{C}_{16} \mathrm{H}_{14} \mathrm{~N}_{2} \mathrm{O}_{4}$.

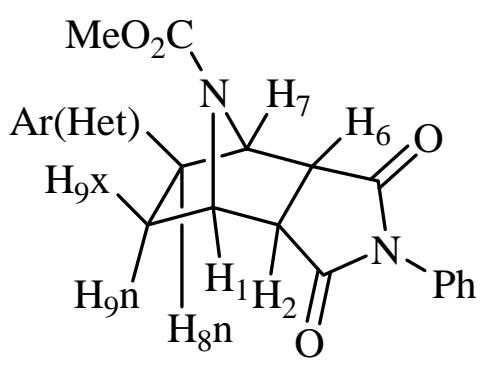

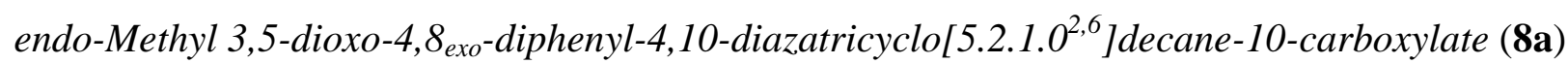

Separated by column chromatography (2:1 ethyl acetate- $n$-hexane) as colorless crystals, yield 55\%; $\mathrm{R}_{\mathrm{f}}$ : 0.49; mp 220-222 ${ }^{\circ} \mathrm{C}$; IR: 3061, 1777, 1713, $1698 \mathrm{~cm}^{-1} ;{ }^{1} \mathrm{H}-\mathrm{NMR} \delta$ : 2.12-2.23 (m, 2H, $\mathrm{H}_{9 \mathrm{x}}$ and $\mathrm{H}_{9 \mathrm{n}}$ ), 3.09-3.26 (m, 2H, $\mathrm{H}_{2}$ and $\mathrm{H}_{6}$ ), 3.46 (s, 3H, $-\mathrm{OCH}_{3}$ ), 3.57 (s, $1 \mathrm{H}, \mathrm{H}_{8 \mathrm{n}}$ ), 4.75 (bs, $1 \mathrm{H}, \mathrm{H}_{1}$ ), 5.06 (bs, 1H, $\mathrm{H}_{7}$ ), 7.21-7.33 (m, 5H, aromatic protons); 7.36-7.48 (m, 5H, aromatic protons); ${ }^{13} \mathrm{C}-\mathrm{NMR} \delta: 37.9,47.7$, 49.3, 50.7, 52.9, 60.6, 65.3, 126.3, 127.2, 128.8, 129.3, 131.9, 142.9, 155.3, 175.2, 175.4; MS: m/z 375 $\left[\mathrm{M}^{+}\right], \mathrm{C}_{22} \mathrm{H}_{20} \mathrm{~N}_{2} \mathrm{O}_{4}$.

endo-Methyl 8-exo-(4-chlorophenyl)-3,5-dioxo-4-phenyl-4,10-diazatricyclo[5.2.1.0 $\left.{ }^{2,6}\right]$ decane-10carboxylate $\mathbf{( 8 b )}$.

Separated by column chromatography (1:1 ethyl acetate- $n$-hexane) as colorless crystals, yield $72 \%$; $\mathrm{R}_{\mathrm{f}}$ : 0.40; mp 182-84 ${ }^{\circ} \mathrm{C}$; IR: 3058, 1776, 1716, $1695 \mathrm{~cm}^{-1} ;{ }^{1} \mathrm{H}-\mathrm{NMR}\left(\delta: 2.07-2.21\left(\mathrm{~m}, 2 \mathrm{H}, \mathrm{H}_{9 \mathrm{x}}\right.\right.$ and $\left.\mathrm{H}_{9 \mathrm{n}}\right)$, 
3.07-3.24 (m, 2H, $\mathrm{H}_{2}$ and $\mathrm{H}_{6}$ ), 3.47 (s, $3 \mathrm{H},-\mathrm{OCH}_{3}$ ), 3.57 (s, $1 \mathrm{H}, \mathrm{H}_{8 \mathrm{n}}$ ), 4.70 (bs, $1 \mathrm{H}, \mathrm{H}_{1}$ ), 5.06 (bs, $1 \mathrm{H}$, $\mathrm{H}_{7}$ ), 7.23-7.29 (m, 5H, aromatic protons), 7.36-7.41 (m, 2H, aromatic protons), 7.46-7.48 (m, 2H, aromatic protons); ${ }^{13} \mathrm{C}-\mathrm{NMR} \delta$ : 37.8, 46.8, 49.0, 50.3, 52.9, 59.2, 65.0, 126.4, 128.3, 128.6, 128.8, 129.1, 131.4, 132.9, 141.2, 155.2, 174.9, 175.0; MS: m/z $410\left[\mathrm{M}^{+}\right], \mathrm{C}_{22} \mathrm{H}_{19} \mathrm{ClN}_{2} \mathrm{O}_{4}$.

endo-Methyl 3,5-dioxo-4-phenyl-8-exo-(2-thienyl)-4,10-diazatricyclo[5.2.1.0 $\left.{ }^{2,6}\right]$ decane-10-carboxylate $(\mathbf{8 c})$.

Separated by column chromatography (1:1 ethyl acetate- $n$-hexane) as colorless crystals, yield 58\%; $\mathrm{R}_{\mathrm{f}}$ : 0.43; mp 224-226 ${ }^{\circ} \mathrm{C}$; IR: 3112, 3064, 1778, 1713, $1691 \mathrm{~cm}^{-1}$; ${ }^{1} \mathrm{H}-\mathrm{NMR} \delta: 2.22-2.24$ (m, $2 \mathrm{H}, \mathrm{H}_{9 \mathrm{x}}$ and $\mathrm{H}_{9 \mathrm{n}}$ ), 3.10-3.12 (dd, $J=7.5,4.0 \mathrm{~Hz}, 1 \mathrm{H}, \mathrm{H}_{6}$ ), 3.21-3.24 (d, $J=9.0 \mathrm{~Hz}, 1 \mathrm{H}, \mathrm{H}_{2}$ ), 3.45 (s, 3H, $-\mathrm{OCH}_{3}$ ), 3.57 (s, $1 \mathrm{H}, \mathrm{H}_{8 \mathrm{n}}$ ), 4.75 (bs, $1 \mathrm{H}, \mathrm{H}_{1}$ ), 5.04 (bs, $1 \mathrm{H}, \mathrm{H}_{7}$ ), 6.92-6.94 (dd, J=4.5, $4.5 \mathrm{~Hz}$, 2H, thienyl protons), 7.24-7.26 (m, $1 \mathrm{H}$, thienyl proton), 7.37-7.49 (m, 5H, aromatic protons); ${ }^{13} \mathrm{C}-\mathrm{NMR} \delta$ : 38.7 , 48.7, 49.6, 52.7, 58.9, 65.8, 124.0, 124.4, 126.0, 126.4, 126.6, 128.8, 129.1, 131.4, 146.2, 155.4, 174.9, 175.0; MS: m/z $382\left[\mathrm{M}^{+}\right], \mathrm{C}_{20} \mathrm{H}_{18} \mathrm{~N}_{2} \mathrm{O}_{4} \mathrm{~S}$.

endo-Methyl 3,5-dioxo-9-exo-(trimethylsilylethynyl)-4,8-exo-diphenyl-4,10-diazatricyclo-[5.2.1.0 $\left.{ }^{2,6}\right]$ decane-10-carboxylate (9).

Separated by column chromatography (1:1 ethyl acetate- $n$-hexane) as yellow crystals, yield 42\%; $\mathrm{R}_{\mathrm{f}}$ : 0.52; mp 196-198 ${ }^{\circ} \mathrm{C}$; IR: 3033, 1781, 1717, $1693 \mathrm{~cm}^{-1}$; ${ }^{1} \mathrm{H}-\mathrm{NMR} \delta: 0.0$ (s, 9H, Si $\left.\left(\mathrm{CH}_{3}\right)_{3}\right)$, 3.313.48 (m, 4H, $\mathrm{H}_{9 \mathrm{n}}, \mathrm{H}_{8 \mathrm{n}}, \mathrm{H}_{2}$ and $\mathrm{H}_{6}$ ), 3.78 (s, 3H, $-\mathrm{OCH}_{3}$ ), 5.08 (bs, $1 \mathrm{H}, \mathrm{H}_{1}$ ), 5.31 (bs, $1 \mathrm{H}, \mathrm{H}_{7}$ ), 7.36-7.45 (m, 5H, aromatic protons), 7.47-7.64 (m, 5H, aromatic protons); ${ }^{13} \mathrm{C}-\mathrm{NMR} \delta: 0.52,42.9,49.0,49.9$, 51.6, 53.0, 63.1, 64.5, 91.6, 102,7, 125.9, 126.0, 127.0, 128.0, 128.3, 128.8, 129.2, 134.2, 139.0, 154.4, 174.3, 174.5; MS: m/z $472\left[\mathrm{M}^{+}\right], \mathrm{C}_{27} \mathrm{H}_{28} \mathrm{SiN}_{2} \mathrm{O}_{4}$.

\section{Acknowledgements}

This research has been supported by the Yıldız Technical University Scientific Research Projects Coordination Department, Project Numbers; 24-01-02-04 and 26-01-02-04. We also thank Marko Spillner and Dr. Jan C. Namyslo, both from the Clausthal University of Technology, for preparing the tricyclic starting material 7 (M.S.) and for some spectroscopic results and fruitful discussions (J.C.N.).

\section{References and Notes}

1. Spande, T. F.; Garraffo, H. M.; Edwards, M. W.; Yeh, H. J. C.; Pannel, L.; Daly, J. W. Epibatidine: A novel (chloropyridyl)azabicycloheptane with potent analgesic activity from an Ecuadoran poison frog. J. Am. Chem. Soc. 1992, 114, 3475-3478.

2. Holladay, M. W.; Dart, M. J.; Lynch, J. K. Neuronal nicotinic acetylcholine receptors as targets for drug discovery. J. Med. Chem. 1997, 40, 4169-4194.

3. Evans, D. A.; Scheidt, K. A.; Downey, C. W. Synthesis of (-)-Epibatidine. Org. Lett. 2001, 3, 3009-3012. 
4. Stuhlmann, F.; Kaufmann, D. E. 2-Nitro derivatives of the alkaloid Epibatidine. J. Prakt. Chem. 1999, 341, 455-460.

5. Ken Lee C. L.; Loh, T. P. Gram-scale synthesis of (-)-Epibatidine. Org. Lett. 2005, 7, 2965-2967.

6. Cox, C., D.; Malpass, J. R. Synthesis of epibatidine isomers: Reductive Heck coupling of 2azabicyclo[2.2.1]hept-5-ene derivatives. Tetrahedron 1999, 55, 11879-11888.

7. Kasyan, A.; Wagner, C.; Maier, M. E. Regiochemistry of the reductive Heck coupling of 2azabicyclo[2.2.1]hept-5-ene. Synthesis of epibatidine analogues. Tetrahedron 1998, 54, 80478054.

8. Larsen, S. D.; Grieco, P. A. Aza-Diels-Alder reactions in aqueous solution: Cyclocondensation of dienes with simple iminium salts generated under Mannich conditions. J. Am. Chem. Soc. 1985, 107, 1768-1769.

9. Drew, M. G. B.; George, A. V.; Isaacs, N. S.; Rzepa, H. S. High-pressure synthesis, structure and conformational properties of some derivatives of 7-azabicyclo[2.2.1]heptane. X-ray determination of endo-10-benzoyl-4-phenyl-4,10-diazatricyclo[5.2.1.0]dec-8-ene-3,5-dione and exo-10-acetyl-4phenyl-4-10-diazatricyclo[5.2.1.0]decane-3,5-dione. J. Chem. Soc. Perkin Trans. 1, 1985, 12771284.

Sample Availability: Samples of the compounds are available from the authors.

(C) 2006 by MDPI (http:www.mdpi.org). Reproduction is permitted for non commercial purposes. 